\title{
O Gepem e seus 37 anos de existência em prol da educação matemática
}

\author{
Rosana de Oliveira \\ Doutora em Educação pela UERJ \\ Professora da EDU/UERJ/PMAR \\ rosanaol@gmail.com
}

\section{Ana Lucia Vaz da Silva \\ Doutora em Educação pela PUC Rio \\ Professora do Colégio Pedro II \\ alvazsilva@hotmail.com}

\section{Andreia Carvalho Maciel Barbosa \\ Doutoranda em Educação Matemática pela UNIBAN \\ Professora do Colégio Pedro II e da FFP/UERJ \\ andreiamaciel@gmail.com}

\section{Dora Soraia Kindel}

Doutora em Educação Matemática pela UNIBAN

Professora da UFRuralRJ

soraiakindel@yahoo.com.br

\section{Resumo}

Nesse relato descrevemos sucintamente o evento realizado na Faculdade de Educação no Campus Maracanã da Universidade do Estado do Rio de Janeiro por ocasião da 46 Assembleia Geral Ordinária do Gepem. No texto podemos observar a importância do Gepem como grupo interinstitucional e como grupo propulsor da área de Educação Matemática.

Palavras-chave: Gepem; Assembleia Ordinária; Evento; Relato de atividades.

\section{The Gepem and its 37 years of existence in behalf of mathematics education}

\begin{abstract}
In this report we describe briefly the event held at the School of Education in the Maracanã Campus of the State University of Rio de Janeiro during the 46th Ordinary General Assembly of Gepem. The text shows the importance of Gepem as an interinstitutional group and as propellant of Mathematics Education area.
\end{abstract}

Keywords: Gepem; Ordinary Assembly; Event; Activities report. 
No dia 23 de setembro de 2013, na Universidade do Estado do Rio de Janeiro, realizou-se mais uma Assembleia Ordinária do Gepem, para realização da contagem dos votos e posse da nova diretoria.

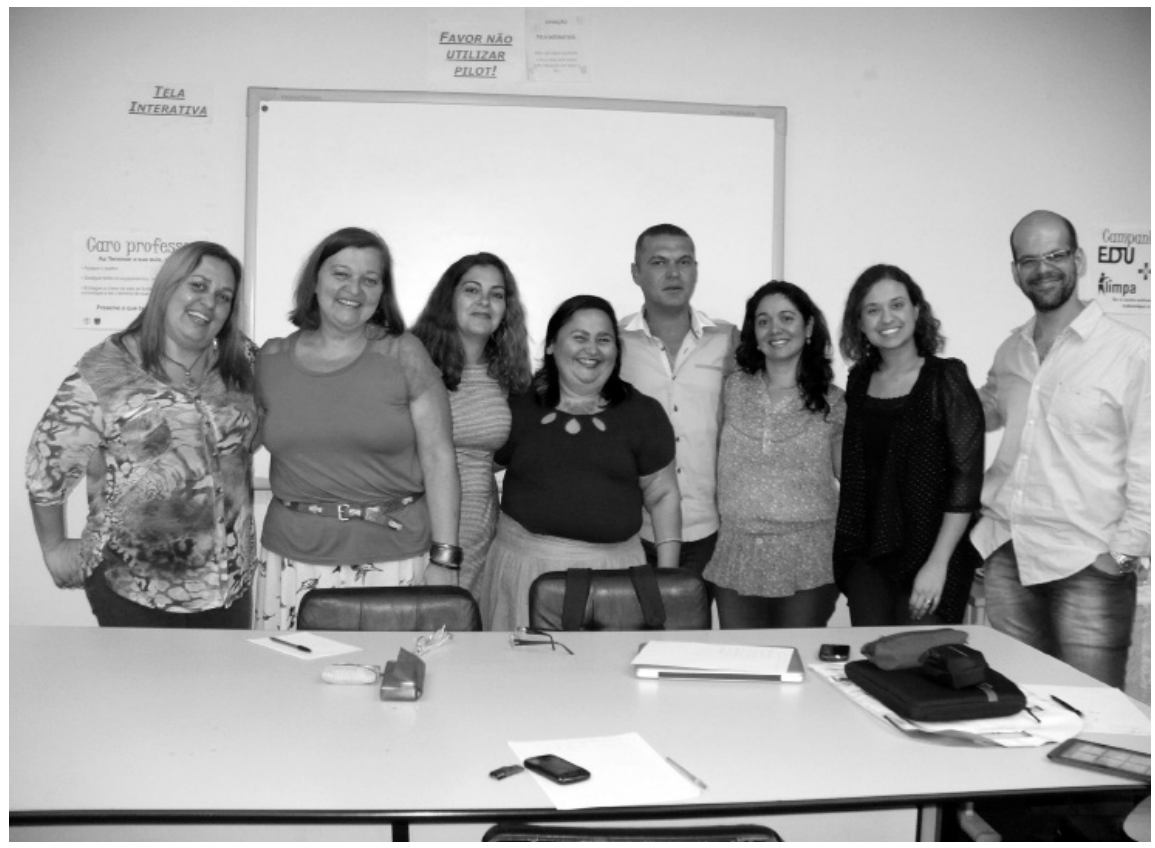

Figura 1: Foto dos participantes da Assembleia. Da esquerda para a direita: Andreia Carvalho Maciel Barbosa, Dora Soraia Kindel, Ana Lucia Vaz da Silva, Rosana de Oliveira, Marcelo Bairral, Neiva Ferreira Alves, Aline Terra Salles e Alexandre Rodrigues de Assis.

O encontro iniciou com um minuto de silêncio em homenagem a professora Maria Laura Leite Lopes Mousinho que este ano nos deixou. A realização de mais uma Assembleia do Gepem é talvez a melhor homenagem que podemos fazer, ou seja, sua realização significa a manutenção do Grupo que ela foi uma das fundadoras e fazia questão de prestigiar em suas falas e artigos.

Nesses anos o Gepem tem conseguido manter-se como um grupo interinstitucional e independente, mas sendo apoiado, de forma significativa, por Instituições de Ensino Superior, originalmente, pela Universidade Santa Úrsula (USU) e, desde 2003, pela Universidade Federal Rural do Rio de Janeiro (UFRRJ).

Além da Assembleia, realizou-se nesse dia a Mesa Redonda intitulada Letramento Matemático: reflexões sobre o papel da Matemática no mundo atual com a participação das professoras Ana Lucia Vaz da Silva (Colégio Pedro II), da professora 
Andreia Carvalho Maciel Barbosa (UERJ/FFP e Colégio Pedro II) e da professora Dora Soraia Kindel (UFRRJ).

A mesa redonda foi aberta pela professora Ana Lucia Vaz da Silva que apresentou um panorama dos conceitos relacionados ao Letramento Matemático, tais como alfabetismo, alfabetismo funcional, letramento, literacia, materacia, numeracia, alfabetismo matemático e numeramento. Fez um paralelo entre as discussões existentes sobre o Letramento, com o uso da escrita e leitura em práticas sociais (GOULART, 2001) e do conceito de numeramento como um amplo conjunto de habilidades, estratégias e ações que o indivíduo necessita para inserir-se autonomamente em situações que envolvem elementos numéricos e conhecimentos matemáticos para resolver problemas da vida quotidiana (PONTE, 2002; TOLEDO, 2003). Destacou, de acordo com D’Ambrósio (2004) a importância de proporcionar aos alunos dos variados segmentos escolares e acadêmicos "uma visão crítica dos instrumentos comunicativos, intelectuais e materiais que eles deverão dominar para que possam viver na civilização que se descortina, vai muito além do ler, escrever e contar". Por fim, indicou as avaliações de larga escala tais como Pisa, Enem, Saeb, Enceja como exemplos práticos escolares do letramento matemático.

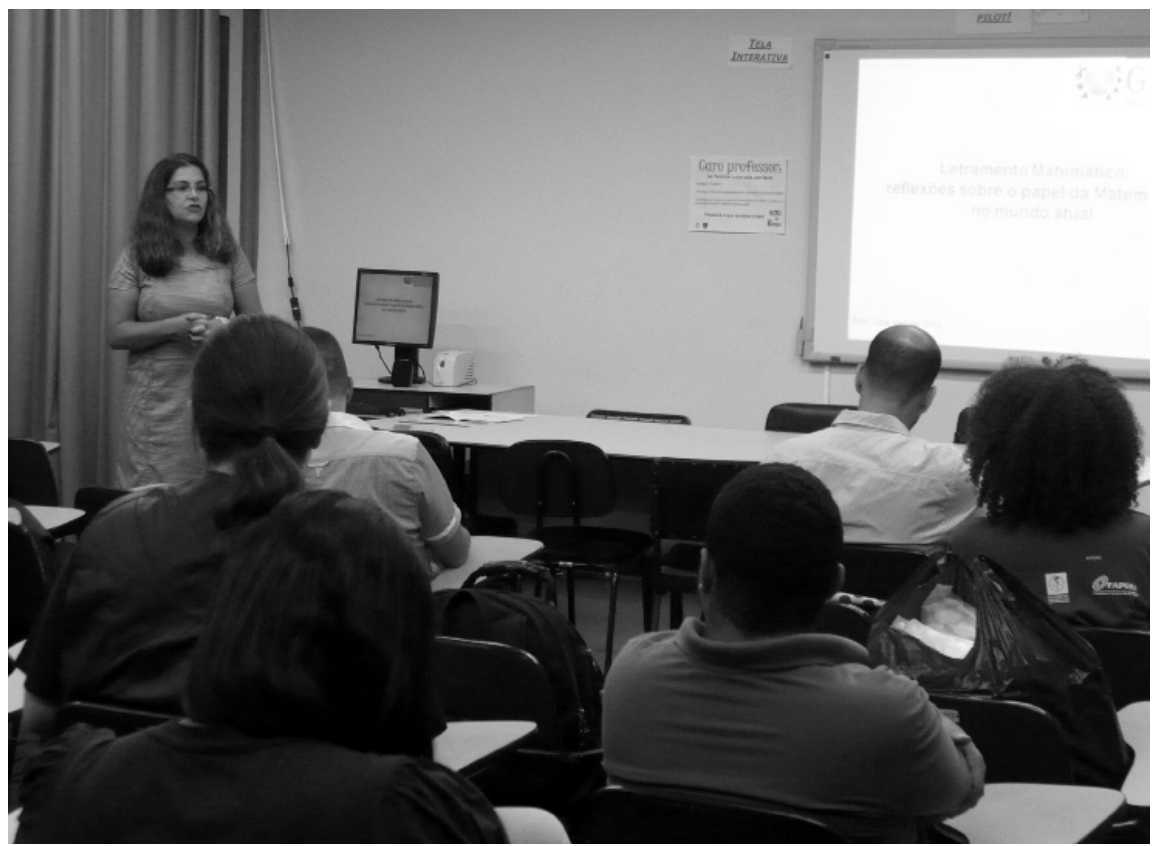

Figura 2: Foto da mesa redonda. Ana Lucia Vaz da Silva apresentando conceitos do letramento matemático. 
No segundo momento, a professora Andreia Carvalho Maciel Barbosa apresentou ideias sobre Letramento Matemático e o uso da Tecnologia em sala de aula. Fez reflexões sobre aparatos tecnológicos existentes para o uso em sala de aula. E contribuiu com exemplos de atividades matemáticas como versões digitais de jogos e materiais manipulativos, tais como o Geoplano e o material dourado e vídeos discutidos a luz da teoria de Bolite Frant e Castro (2011) que classificam o uso da tecnologia em ferramenta, meio de expressão e prótese. Para concluir, apresentou ideias do educador matemático Ubiratan D’Ambrosio apresentadas durante o Congresso Iberoamericano de Educação Matemática 2013, no qual sinalizou que o uso da tecnologia faz parte dos desafios do mundo em que vivemos e que cabe aos educadores tomarem a iniciativa, de acordo com suas possibilidades, propondo novas direções que possibilitem aos alunos a compreender, explicar e agir no mundo atual.

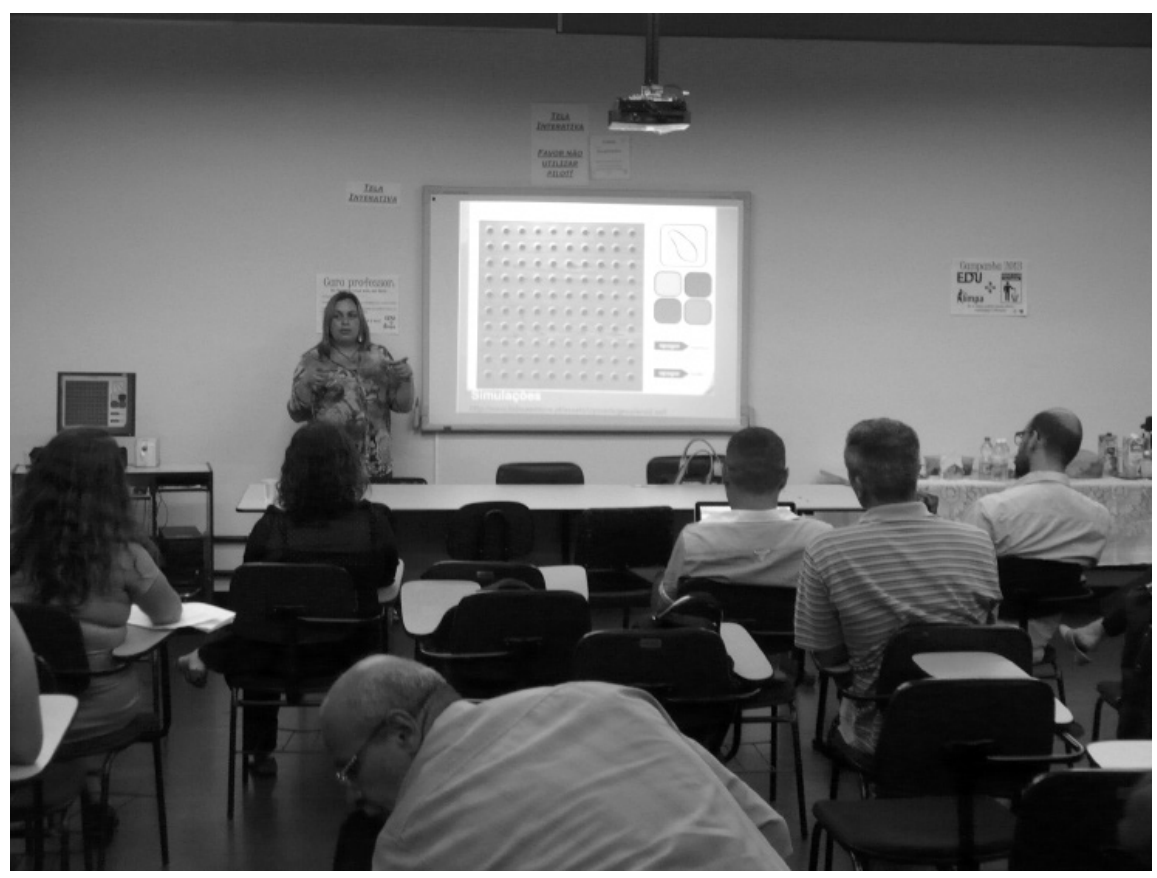

Figura 3: Foto da mesa redonda. Andreia Carvalho Maciel Barbosa, apresentando reflexões sobre o uso da tecnologia no letramento matemático.

A professora Dora Soraia Kindel inicia sua apresentação com uma reflexão importante sobre a Matemática ser uma atividade individual ou uma prática coletiva? 
Com foco no desenvolvimento cognitivo e na aprendizagem acadêmica, afirma que a década de 1970 caracteriza-se pela "morte e renascimento do campo da aprendizagem da criança" (SIEGLER apud GOLBERT, p. 7, 2011), com mudanças na forma que se entende o papel das crianças e das tarefas propostas para a aprendizagem. Teorias como o Neuroconstrutivismo e a Neurociência Cognitiva, confirmam que cognição não é um estado e sim um processo em permanente construção. A ênfase nas fases de desenvolvimento cognitivo ou na aprendizagem cognitiva pode causar distorções no processo de construção do conhecimento, para dialogar com essas posições, a cultura e a linguagem se constituem como elementos relevantes. A professora finaliza sua apresentação com uma importante contribuição sobre o que acredita ser sua função como professora em sala de aula, afirmando que: "eu não preparo aula, eu me preparo para aula" e conclui que "os alunos são extremamente criativos e curiosos e isso deve ser preservado".

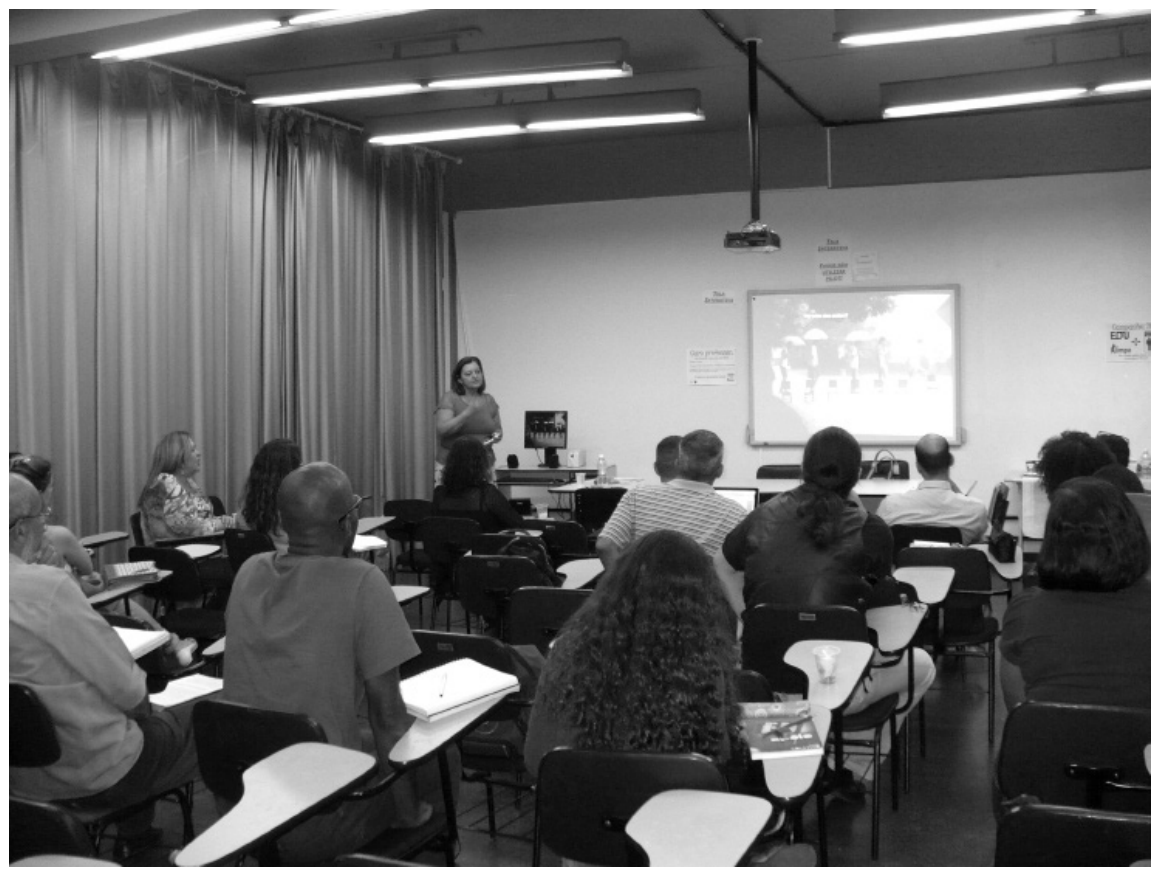

Figura 4: Foto da mesa redonda. Dora Soraia Kindel apresentando reflexões sobre o papel da atividade no letramento matemático.

O evento contou com a participação cerca de 40 alunos do Curso de Pedagogia da Faculdade de Educação da UERJ, gerando um rico debate entre os alunos 
e as professoras palestrantes. Entre os temas abordados, vale destacar que o uso da calculadora nos anos iniciais ainda gera dúvidas sobre sua utilização em sala de aula, em particular para os licenciandos em Pedagogia.

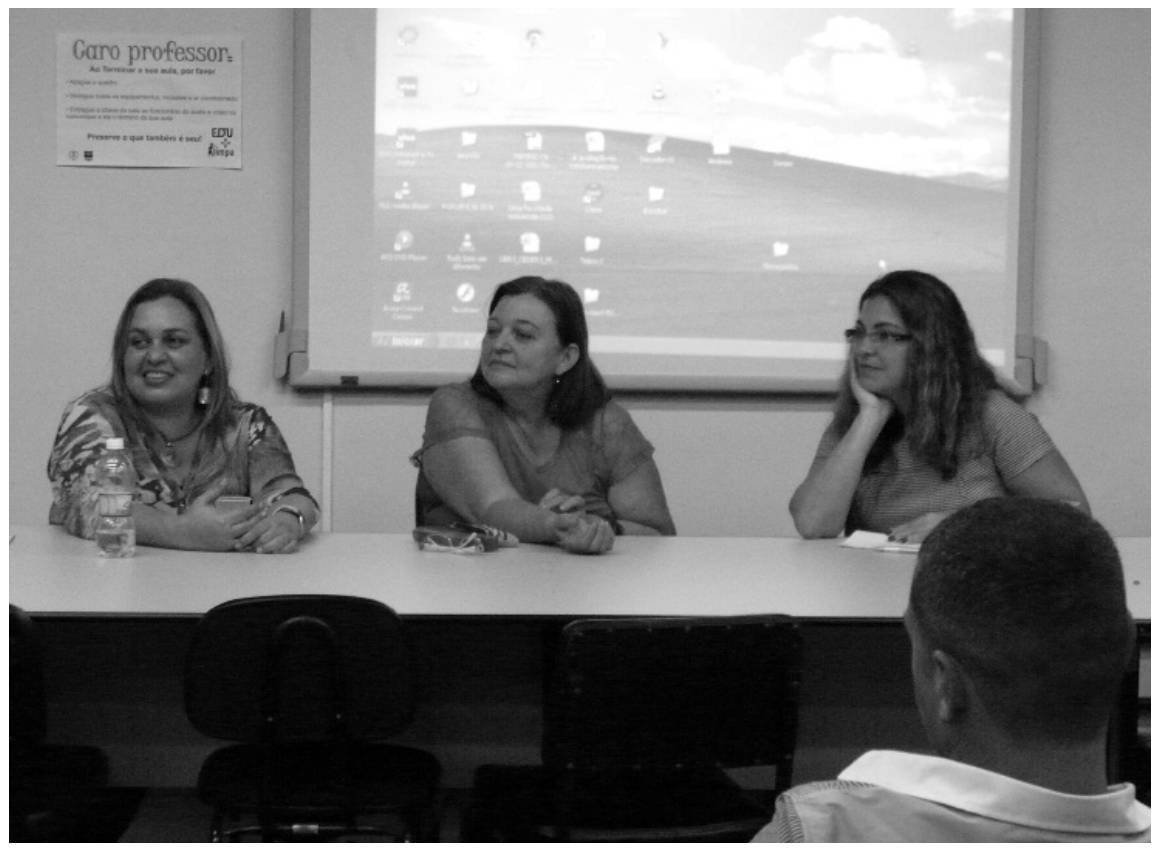

Figura 5: Foto da mesa redonda - debate. Da esquerda para a direita: Andreia Carvalho Maciel Barbosa, Dora Soraia Kindel, Ana Lucia Vaz da Silva,

A seguir apresentamos a Chapa eleita e sua proposta de trabalho para o Biênio 2013-2015.

Chapa eleita para a Gestão do GEPEM no Biênio 2013-2015 Presidente: Marcelo Almeida Bairral

Doutor em Educação Matemática, Professor da UFRRJ/IE/DTPE.

\section{Vice-Presidente: Dora Soraia Kindel}

Doutor em Educação Matemática, Professora da UFRRJ/IM/DES.

\section{Secretária Geral: Aline Terra Salles}

Mestranda em Educação (UFRRJ) e Professora substituta da UERJ (Maracanã). 
Segundo Secretário: Alexandre Rodrigues de Assis

Professor do Instituto de Educação Rangel Pestana (Nova Iguaçu/RJ).

\section{Primeiro Tesoureiro: Márcio de Albuquerque Vianna}

Doutorando em Ciência, Tecnologia e Inovação, Professor da UFRRJ/IE/DTPE.

\section{Segundo Tesoureiro: Wagner da Silveira Marques}

Mestrando em Educação (UFRRJ), Professor da FAETEC (Santa Cruz, RJ) e da Universidade Cândido Mendes.

\section{Diretora Cultural: Neiva Ferreira Alves}

Especialista em Educação Matemática, Professora da Rede Estadual do Rio de Janeiro.

\section{Comitê Editorial}

Ana Lucia Vaz da Silva (Colégio Pedro II)

Andreia Carvalho Maciel Barbosa (Colégio Pedro II e UERJ/FFP)

Dora Soraia Kindel (UFRRJ)

Marcelo Almeida Bairral (UFRRJ)

Rosa Maria Mazo Reis (UNESA)

Rosana de Oliveira (UERJ, SMAR)

\section{Proposta de trabalho, 2013-2015}

1. Publicar a versão impressa do Boletim Gepem 63 (jul./dez. 2013).

2. Manter a publicação semestral do Boletim GEPEM, porém apenas na sua versão online.

3. Continuar trabalhando para melhorar a qualificação do Boletim Gepem na avaliação de periódicos da Capes.

4. Continuar trabalhando com a inserção do Boletim Gepem em bases e indexadores importantes, como o Scielo e a ISIS.

5. Finalizar a disponibilização online (gratuita) dos Boletins impressos.

6. Continuar ampliando o número de textos publicados no Boletim Gepem e criar o DOI para os mesmos.

7. Incentivar a publicação de números temáticos (como fascículos complementares ou regulares) do Boletim Gepem.

8. Condicionar à submissão de textos ao Boletim Gepem a inscrição ou atualização da anuidade corrente ao Gepem pelo autor e coautores da submissão. 
9. Manter atualizado o Portal do GEPEM na Internet.

10. Manter o Banco de Dados de sócios atualizado.

11. Viabilizar a publicação de livros - desde que sejam submetidos e aprovados pelo comitê editorial do GEPEM e que existam recursos disponíveis - de nossos associados, dando continuidade a Série Pensamento em Ação.

12. Continuar estabelecendo intercâmbio com grupos de pesquisa ou Instituições de Ensino e associações (nacionais e internacionais) da área de Educação Matemática ou afins.

13. Continuar divulgando o GEPEM junto a Secretarias (Municipais/Estaduais) de Educação visando a associar professores, divulgar as publicações do GEPEM e auxiliar na realização de eventos.

14. Participar de encontros locais, regionais, nacionais e internacionais de Educação Matemática divulgando o GEPEM.

15. Incentivar a realização de eventos para a formação (inicial ou continuada) de professores.

16. Prestar relatório e renovar o convênio do Gepem com a UFRRJ.

\section{Referências}

BOLITE FRANT, J.; CASTRO, M. R. O modelo da estratégia argumentativa: análise da fala e de outros registros em contextos interativos de aprendizagem. Ed 1. Curitiba: UFPR, 2011.

D’AMBRÓSIO, U. A relevância do projeto Indicador Nacional de Alfabetismo Funcional - INAF - como critério de avaliação da qualidade do ensino de matemática. In: FONSECA, M. C. F. R. (Org.). Letramento no Brasil: habilidades matemáticas. São Paulo: Global, 2004. p. 31-46.

GOLBERT, C. S. Matemática nas séries iniciais: o sistema decimal de numeração. Porto Alegre: Mediação, 2011.

GOULART, C. Letramento e polifonia: um estudo de aspectos discursivos do processo de alfabetização. Revista Brasileira de Educação, n. 18, set./dez. 2001.p. 5-21.

PONTE, J. P. Literacia matemática. In M. N. Trindade (Org). Actas do Encontro Internacional Literacia e cidadania: Convergências e interfaces (em CD-ROM). Universidade de Évora: Centro de Investigação em Educação Paulo Freire, 2002. http://www.educ.fc.ul.pt/docentes/jponte/artigos_pt.htm 
SIEGLER, R. S. Children's Learning. American Psycologist, n.60. 2005. p. 769-778.

TOLEDO, M. E. R. O. Numeramento e escolarização: o papel da escola no enfrentamento das demandas matemáticas cotidianas. In: FONSECA, M. C. F. R. (Org.). Letramento no Brasil: habilidades matemáticas. São Paulo: Global, 2004. p. 91-105.

Texto encomendado Submetido em outubro de 2013 